\title{
SETS OF CONVERGENCE OF EXPONENTIAL SERIES
}

\author{
DON R. LICK ${ }^{1}$
}

Brauer [1, Research Problems] suggested the following problem. Let $\left\{\alpha_{n}\right\}$ denote a sequence of real numbers contained in the interval $[0,2 \pi]$ and dense in a subinterval of length $\pi / 2$. If $E$ is a given subset of the set of natural numbers, does there exist a series of the form

$$
\sum_{n=1}^{\infty} c_{n} \exp \left(i k \alpha_{n}\right)
$$

which converges when the natural number $k$ is in $E$ and diverges when the natural number $k$ is not in $E$ ? In this note we prove the following, which contains, among other things, a partial answer to Brauer's question.

THEOREM. Let $\left\{\alpha_{n}\right\}$ denote a sequence of numbers contained in the interval $[0,2 \pi]$ and dense in a subinterval of length $\eta$ for some positive number $\eta$. Let $J(\eta)$ denote the set of integers which are divisible by $[2 \pi / \eta]$ where the symbol $[x]$ denotes the largest positive integer not greater than $x$. If $M(\eta)$ is a prescribed subset of $J(\eta)$ then there exists a series (1) that diverges for each integer $k$ in $M(\eta)$ and converges for each integer $k$ in $J(\eta)-M(\eta)$.

In order to prove this theorem, we need the following two lemmas.

Lemma. Let $M$ be any set of integers. Then there exists a sequence $\left\{\lambda_{n}\right\}$ of real numbers tending to infinity, and a series

$$
\sum_{n=1}^{\infty} c_{n} \exp \left(i k \lambda_{n}\right)
$$

that diverges for each integer $k$ in $M$ and converges for each integer not in $M$, and which has uniformly bounded partial sums; $\left\{\lambda_{n}\right\}$ does not depend on $M$.

We first consider the case where $M=\{0\}$. Let

$$
\begin{gathered}
F_{n}(k)=1 / n+\exp (i k) /(n-1)+\cdots+\exp ((n-1) i k) / 1 \\
-\exp (n i k) / 1-\cdots-\exp ((2 n-1) i k) / n
\end{gathered}
$$

be the $n$th Fejér trigonometric polynomial. Let

Received by the editors March 24, 1967 and, in revised form, May 1, 1967.

I The author would like to thank George Brauer for his many helpful suggestions. 


$$
\begin{aligned}
G_{p}(k) & =\left(\log h_{p}\right)^{-1}\left(\exp \left(n_{p} i k / H_{p}\right)\right) F_{h_{p}}\left(k / H_{p}\right) \\
& =\sum_{r=0}^{2 h_{p}-1} a_{p, r} \exp \left(\left(n_{p}+r\right) i k / H_{p}\right),
\end{aligned}
$$

where $h_{p}$ and $H_{p}$ are positive in tegers; the $n_{p}$ are positive real numbers chosen to ensure nonoverlapping of the trigonometric polynomials (4) (for example, $n_{p+1} / H_{p+1}>n_{p} / H_{p}+2 h_{p} / H_{p}$ ); and

$$
\begin{aligned}
a_{p, r} & =1 /\left(h_{p}-r\right)\left(\log h_{p}\right), \quad \text { if } 0 \leqq r<h_{p}, \\
& =-1 /\left(r-h_{p}+1\right)\left(\log h_{p}\right), \quad \text { if } h_{p} \leqq r<2 h_{p} .
\end{aligned}
$$

Now if $\left\{n_{p} / H_{p}\right\}$ tends to infinity, the series

$$
\sum_{p=1}^{\infty} G_{p}(k)
$$

will constitute an exponential series of the form (2). Since the Fejér polynomials (3) are uniformly bounded by an absolute constant $A$ (see [2, pp. 42-43]), $\left|G_{p}(k)\right|<A /\left(\log h_{p}\right)$, and if $\sum\left(\log h_{p}\right)^{-1}$ converges, the series (5) converges absolutely for any integer $k$.

By the method used in [3, p. 263] the series (2) defined by the series (5) can be shown to diverge for $k=0$ and to converge for all other values of $k$; furthermore, this series has uniformly bounded partial sums.

In the general case where $M=\left\{m_{q}: q=1,2, \cdots\right\}$, let

$$
\begin{aligned}
G_{p, q}(k) & =\left(\log h_{p}\right)^{-1}\left(\exp \left(n_{p, q} i k / H_{p}\right)\right) F_{h_{p}}\left(\left(k-m_{q}\right) / H_{p}\right) \\
& =\sum_{r=0}^{2 k_{p}-1} a_{p, q, r} \exp \left(\left(n_{p, q}+r\right) i k / H_{p}\right),
\end{aligned}
$$

where the $n_{p, q}$ are positive numbers chosen to prevent overlapping of the trigonometric polynomials $G_{p, q}(k)$ and

$$
\begin{aligned}
a_{p, q, r} & =\exp \left(-i r m_{q} / H_{p}\right) /\left(h_{p}-r\right)\left(\log h_{p}\right), \quad \text { if } 0 \leqq r<h_{p}, \\
& =-\exp \left(-i r m_{q} / H_{p}\right) /\left(r-h_{p}+1\right)\left(\log h_{p}\right), \quad \text { if } h_{p} \leqq r<2 h_{p} .
\end{aligned}
$$

Then the series $\sum_{p, q} 2^{-q} G_{p, q}(k)$ constitutes an exponential series of type (2) with the desired properties.

Lemмa. If $M(\eta)$ is a given set of integers in $J(\eta)$, then there exists a sequence of numbers $\left\{\beta_{n}\right\}$ in $[0, \eta]$ and a series $\sum_{n=1}^{\infty} c_{n} \exp \left(i k \beta_{n}\right)$ which diverges when $k$ is in $M(\eta)$ and converges when $k$ is $J(\eta)-M(\eta)$; the sequence $\left\{\beta_{n}\right\}$ is independent of $M(\eta)$. The series has uniformly bounded partial sums. 
Let $\left\{H_{p}\right\}$ be any sequence of positive integers that tends to infinity. Let $\left\{h_{p}\right\}$ be a sequence of positive in tegers such that $\sum\left(\log h_{p}\right)^{-1}$ converges and the sequence $\left\{H_{p} /\left(\log h_{p}\right)\right\}$ tends to zero. Let $\left\{n_{p, q}\right\}$ be a sequence of positive numbers such that

$$
n_{p, q}=2 \pi H_{p} N_{p, q} /[2 \pi / \eta],
$$

where $\left\{N_{p, q}\right\}$ is a sequence of positive integers and the $n_{p, q}$ satisfy the conditions in the above proof. Then

$$
\begin{aligned}
\exp \left(\left(n_{p, q}+r\right) i k / H_{p}\right) & =\exp \left\{\left[2 \pi N_{p, q} /[2 \pi / \eta]+r / H_{p}\right] i k\right\} \\
& =\exp \left(r i k / H_{p}\right)
\end{aligned}
$$

for all $k$ in $J(\eta)$. Let

$$
\beta_{p, r}=\left(r / H_{p}\right) \bmod (2 \pi /[2 \pi / \eta]) .
$$

We may write

$$
G_{p, q}(k)=\sum_{r=0}^{2 h_{p}-1} a_{p, q, r} \exp \left(i k \beta_{p, r}\right)
$$

and the series

$$
\sum_{p, q} 2^{-q} G_{p, q}(k)=\sum_{n=1}^{\infty} c_{n} \exp \left(i k \beta_{n}\right)
$$

has the desired properties.

We now return to the proof of our theorem. Without loss in generality we may assume that the numbers $\left\{\alpha_{n}\right\}$ are dense in the interval $[0, \eta]$. Since the numbers $\left\{\beta_{n}\right\}$ are contained in the interval $[0, \eta]$ we may choose for each $\beta_{n}$ a number $\alpha_{m_{n}}$ which is close enough to $\beta_{n}$ for the series

$$
\sum_{n=1}^{\infty} c_{n}\left[\exp \left(i k \alpha_{m_{n}}\right)-\exp \left(i k \beta_{n}\right)\right]
$$

to converge (though not uniformly). With this choice of exponents, which are in the given set $\left\{\alpha_{n}\right\}$, we have constructed a series of the form (1) with the desired properties. We note that the series that we have constructed no longer has uniformly bounded partial sums.

Finally we should note that our result cannot be improved very much. If the exponents $\left\{\alpha_{n}\right\}$ which we are permitted to use lie in an interval $[0, \eta], \eta<\pi / 2$, the behavior of the series (1) at some number $k_{0}$ rules the behavior at adjacent numbers in that divergence of the series at $k_{0}$ forces divergence for $k_{0} \leqq k<k_{0}+[\pi / 2 \eta]$. 


\section{REFERENCES}

1. G. Brauer, Sets of convergence of exponents series, Research Problem, Bull. Amer. Math. Soc. 72 (1966), p. 470.

2. P. Erdös, F. Herzog and G. Piranian, On Taylor series of functions regular in Gaier regions, Arch. Math. 5 (1954), 39-52.

3. - Sets of divergence of Taylor series and of trigonometric series, Math. Scand. 2 (1954), 262-266.

WESTERN Michigan UNIVERSITy 